innerhalb des Gemeindebanns und der nächsten Nachbarschaft ermöglicht den Bewohnern der Rarner Schattenberge ihr Leben. Ohne die niederschlagsreichen Höhen der Felsenkämme, die das Wasser für die Wiesen spenden, und ohne die nur zwei Monate im Jahr beweideten Alpen könnte das Vieh, die Hauptlebensgrundlage der Landschaft, nicht durchgefüttert werden. Ohne die trockene Wärme der unteren Berghänge und der Terrasse mit den Dörfern mangelte das tägliche Brot und die Lebenswürze des Weins. Unten im Rhonetal liegen, durch Verkehr, Wasserkraft und billige Arbeitskräfte begünstigt, die Fabriken der Lonza-Werke, die vielen Bewohnern der drei Gemeinden den Lebensunterhalt bieten.

Besonders bezeichnend für die Rarner Schattenberge ist:

1. Die Viehzucht ist nur möglich auf Grund der Bewässerung der Wiesen, denen die Suonen an den Berghängen das Wasser zuführen.

2. Das Wirtschaftsland einer jeden Familie ist auf verschiedene Höhengürtel verteilt, von den Weinbergen unten im Rhonetal bis hinauf zu den Alpweiden. Das macht im Laufe des Jahres viele Wanderungen der ganzen Familie oder einzelner Familienglieder nötig. Deshalb besitzt jede Familie an verschiedenen Orten, selbst unten im Rhonetal, eine Wohnstätte.

3. Die Hauptsiedelungen, die Kirchdörfer, liegen auf der Terrassenfläche, welche schon in gallorömischer Zeit, früher als die Rhonetalsohle, besiedelt war. Die wirtschaftlichen Wanderungen gehen daher nicht nur aufwärts, sondern auch abwärts ins Rhonetal.

4. Die Rarner Schattenberge sind mit Ergisch ob Turtmann der westlichste Teil des durch die Alemannen von Osten her, über die Grimsel, deutschsprachig besiedelten Oberwallis. Das schluchtartige, bewaldete Turtmanntal, das auch heute nur Alpweiden, keine Dauersiedlungen hat, bildet die Sprach- und Kulturgrenze gegen das Val d'Anniviers und das welsche Wallis.

\title{
LES DÉPOTS QUATERNAIRES ET LA THÉORIE DES EMBOITEMENTS
}

Avec 4 figures

Par ADRIEN JAYET

Introduction

La physionomie, si particulière, du Plateau suisse est due, pour une bonne part à la présence des dépôts quaternaires et aux érosions que ces dépôts ont subies. Depuis plus de cent ans, ils ont fait l'objet d'études minutieuses publiées dans de nombreuses revues scientifiques ou dans des travaux de grande envergure. Citons l'œuvre classique: «Die Alpen im Eiszeitalter» de A. PENCK et E. BrüCKNER ou encore l'article «Diluvium» dans la "Geologie der Schweiz» de Ax. Herm. D'autre part, on trouvera une vue d'ensemble du Quaternaire suisse dans l'important travail de P. BECK: "Ueber das schweizerische und europäische Pleistozän»; une liste bibliographique complète est donnée dans le traité de M. Gignoux: "Géologie stratigraphique». Enfin les différents travaux de M. MrLANKowitch fournissent aux partisans des glaciations multiples, l'appui de données astronomiques; nous indiquons dans la liste bibliographique le dernier de ces travaux.

Il semblerait alors que le Quaternaire soit l'une des périodes les mieux connues de celles qui occupent les géologues. Il n'en est rien, bien au contraire; au fur et à mesure de l'apparition des nouveaux résultats que la glaciologie, la stratigraphie, la paléonto- 
logie, la préhistoire apportent à cette étude, des divergences de plus en plus marquées se font jour dans les interprétations. C'est ainsi que le nombre des glaciations est toujours fortement discuté. Pour certains, il n'y en a qu'une; pour d'autres deux, enfin d'autres encore en voient quatre ou plus, jusqu'à douze. De telles divergences marquent bien aussi qu'il y a des différences dans les méthodes employées. Il s'agit donc, pour s'entendre, de préciser l'emploi de ces méthodes et d'analyser de façon critique les conclusions qu'elles entraînent. C'est un exemple de critique de méthode que je voudrais exposer ici, en montrant, qu'à la méthode analytique de la morphologie doit s'adjoindre un examen très poussé des dépôts que l'on veut interpréter. Mais je dois me borner au strict essentiel; c'est pourquoi on voudra bien consulter, pour de plus amples détails, les trois notes que j'ai récemment consacrées à l'interprétation du Quaternaire régional: Origine et âge de l'alluvion ancienne des environs de Genève; A propos de l'âge du maximum glaciaire quaternaire; Glaciologie quaternaire et Préhistoire.

\section{La notion classique des emboîtements}

Rappelons ce qu'est, dans ses grandes lignes, la théorie des emboîtements. On sait qu'un cours d'eau dépose, pendant certaines phases de son existence et sur certains points de son tracé, des matériaux alluvionnaires; la taille de ces derniers dépend de la vitesse du courant au point où s'est fait l'alluvionnement. La superposition plus ou moins continue des alluvions a donné une terrasse; plus tard par suite de l'abaissement du niveau de base ou ce qui revient au même, d'un exhaussement du sol à l'amont, le cours d'eau attaque la nappe d'alluvions précédemment formée et donne par suite une nouvelle terrasse (fig. 1). La nouvelle terrasse est ainsi emboîtée dans la première. De nouveaux encaissements donnent de nouveaux emboîtements et l'on a finalement une succession de terrasses.

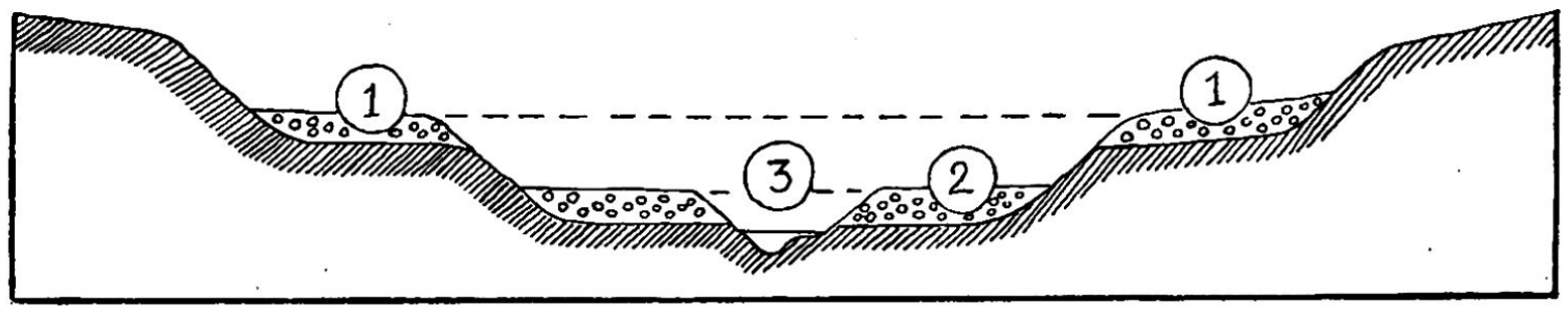

Fig. 1. Coupc à travers une vallée montrant deux systèmes de terrasses emboîtées. 1 Dépôts gravcleux et sableux de la terrasse la plus élevée 2 Dépöts de la terrasse la plus récente. 3 Cours d'eau actuel. Les emboítements de ce type correspondent aux diff́rentes phases du retrail glaciaire. Comparer avec la figure 4 .

On a repéré, un peu partout en Suisse, des niveaux alluvionnaires emboîtés les uns dans les autres. Ils sont particulièrement nets dans la région du Rhin et de ses affluents. La détermination de l'âge relatif de ces nappes alluvionnaires est facile; plus les nappes d'alluvions sont élevées, plus elles sont anciennes.

Cas de l'emboîtement des nappes alluvionnaires en relation avec des moraines glaciaires

Le cas simple de l'emboîtement des nappes alluvionnaires se complique en Suisse par le fait qu'elles se trouvent souvent en relation avec des moraines glaciaires. On admet que les nappes d'alluvions se développent à l'aval des moraines frontales auxquelles elles se raccordent, le tout constituant un complexe fluvio-glaciaire, suivant le schéma bien connu de A. PENCK. Il va de soi que deux périodes glaciaires pourront montrer deux complexes fluvio-glaciaires emboîtés, les restes de la plus ancienne de ces glaciations pouvant aussi correspondre aux dépôts les plus élevés. 
Dans le cas des quatre glaciations de A. PeNCK (Günz, Mindel, Riss, Würm) on retrouve aussi des emboîtements, mais le maximum glaciaire est le fait d'une seule de ces glaciations, celle du Riss d'après les idées en cours, celle du Würm, d'après mes constatations personnelles.

\section{Relations entre une nappe alluvionnaire et une moraine glaciaire, cas du complexe würmien}

C'est ici que mes observations personnelles sont en désaccord avec les idées classiques. Dans la région du Léman et du Rhône, les dépôts quaternaires les plus importants sont ceux du Würm, c'est-à-dire ceux de la dernière glaciation. La figure 2 donne une idée de la superposition de ces dépôts. La remarque essentielle est que la nappe de graviers de l'alluvion ancienne ne se raccorde pas latéralement avec des moraines frontales, mais qu'il y a, au contraire, passage vertical ménagé de cette alluvion à la moraine de fond qui lui est superposée.

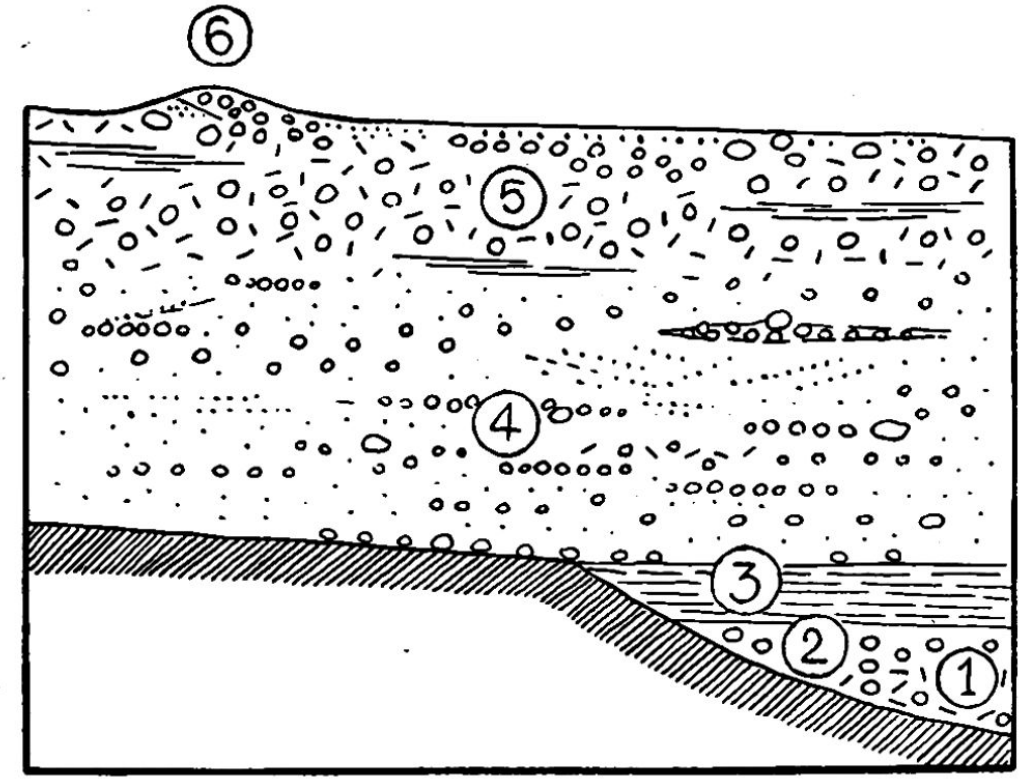

Fig. 2. Coupe demi-schématique des dépôts quaternaires de la région genevoise. 1 Moraine de fond rissienne. 2 Graviers du retrait rissien. 3 Argiles stratifiées interglaciaires à lignitc. 4 Alluvion ancienne de la phase de transgression würmiennc. 5 Moraine de fond du maximum glaciaire würmicn. 6 Moraines et graviers de la phase de régression würmicnne ou retrait. Le complexe würmien, avec ses trois phases, correspond aux dépóts 4, 5, 6 .

La description détaillée de l'alluvion ancienne se trouve dans de nombreux travaux géologiques sur la région de Genève et du Léman. On pense généralement qu'elle est formée de graviers fluvio-glaciaires déposés à l'aval du glacier thodanien en crue. Mais cette opinion ne paraît pas tenir compte de certains caractères, peu apparents à première vue, mais très importants pour l'interprétation de l'âge et de l'origine du dépôt. C'est ainsi qu'au milieu de sables et de graviers plus ou moins grossiers et toujours roulés, apparaissent quelquefois de gros blocs, des lames d'argiles à blocaux, des galets striés; en outre, en plusieurs points on voit un passage progressif de l'alluvion à la moraine de fond qui lui est superposée. Dans les caractères négatifs, il faut noter l'absence de laisses à mollusques, l'absence générale d'un ancien sol au contact avec la moraine de fond, l'absence des affouillements qui auraient dû se produire lors du déplacement latéral du cours d'eau. On est ainsi amené à l'idée que l'alluvion ancienne n'est pas un dépôt fluviatile ordinaire, j'y vois une alluvion sous-glaciaire et j'ai proposé de la désigner par les termes d'alluvion de transgression würmienne pour indiquer qu'elle a été déposée sous le glacier würmien en crue.

Le complexe würmien est par conséquent constitué par trois phases: celle de la transgression marquée par l'alluvion ancienne, celle du maximum au cours de laquelle 
se dépose la moraine de fond, enfin par une phase de régression ou retrait qui comprend les graviers morainiques, souvent en traînées margino-frontales bien reconnaissables, ainsi que de grandes nappes d'alluvions. C'est à ce moment seulement que les encaissements prévus par la loi des emboîtements se produisent et que l'on peut alors parler de terrasses emboîtées.
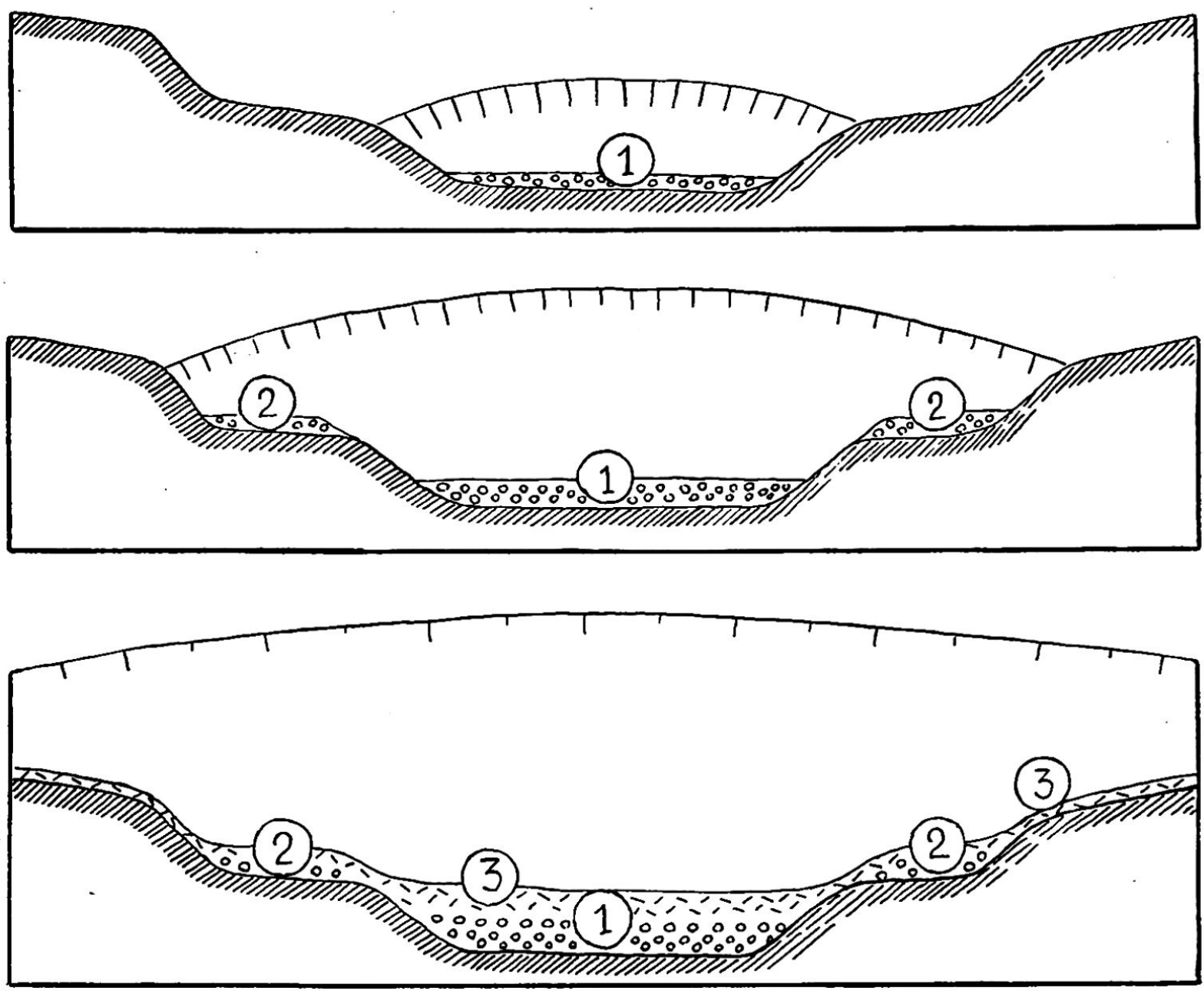

Fig. 3. Représentation schématique de la succession des dépôte lors de l'avance glaciaire würmienne. 1 Graviers sous-glaciaires (alluvion ancienne) déposés dans le thalweg. 2 Graviers sous-glaciaires (alluvion ancienne de type ordinaire et graviers de typé plus morainique) déposés lors de l'accroissement du glacier en épaisseur et en largeur. 3 Moraine de fond correspondant à la phase du maximum. Remarque. L'extension glaciairc du Riss, précédant celle du Würm, n'a pas atteint la région figuréc par cette coupe.

Dépôts alluvionnaires à des altitudes croissantes, pseudo-emboîtements

Si l'on s'écarte du sillon du Léman-Rhône, on constate que l'alluvion ancienne s'élève de plus en plus. Alors que le contact alluvion-moraine est à $400 \mathrm{~m}$. d'altitude aux environs de Genève, à 440 à $450 \mathrm{~m}$. sur les coteaux molassiques de Pregny et de Cologny, à $480 \mathrm{~m}$. à la Châtaigneraie au-dessus de Coppet, elle atteint une altitude de plus de $700 \mathrm{~m}$. dans la falaise de la Côte. Dans certaines parties de cette région; elle contient une accumulation de gros blocs qui lui confère manifestement l'allure d'une moraine latérale; partout elle est recouverte d'un manteau plus ou moins épais de moraine de fond. Si l'on veut lui appliquer la théorie des emboîtements, il faudrait alors considérer les parties les plus élevées comme les plus anciennes, mais l'inverse est beaucoup plus probable.

Si l'on veut bien voir dans l'alluvion ancienne un dépôt sous-glaciaire, il faut alors admettre que les basses parties ont d'abord été atteintes et recouvertes (Léman-Rhône), 
peut-être d'une façon discontinue. Quand le glacier augmente d'épaisseur et de largeur, les collines sont aussi recouvertes, enfin plus tard, les régions élevées sont atteintes à leur tour... Avec l'augmentation d'épaisseur du glacier, la nature du dépôt change et l'on passe des graviers de l'alluvion à la moraine de fond riche en argile et en galets striés. J'essaie de donner une interprétation de ces phénomènes dans la figure 3 . Les dépôts de l'alluvion ancienne ne sont donc pas emboîtés de haut en bas comme on pourrait l'admettre, mais bien de bas en haut, il s'agit donc d'un pseudo-emboîtement.

Lors du retrait glaciaire, les dépôts morainiques graveleux et les terrasses se forment bien suivant la théorie des emboîtements encore ne faut-il pas surestimer les différences chronologiques que peut représenter la succession de ces phénomènes, telle que la représente la figure 4.

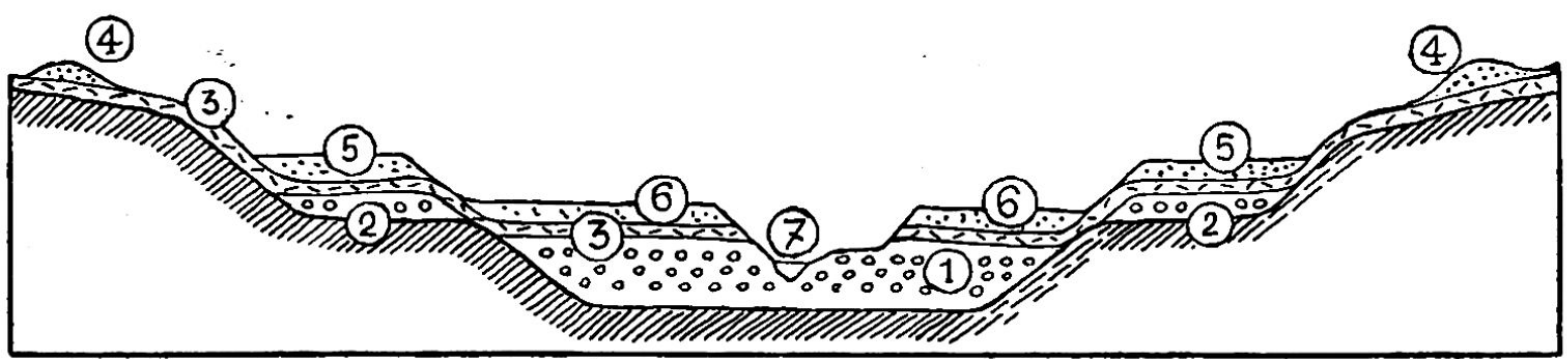

Fig. 4. Coupe transversale schématique d'une vallée du plateau suisse montrant l'allure des dépôts après le retrait glaciaire et la répartition des éléments du complexe würmien. 1 et 2 Graviers de l'alluvion ancienne. 3 Moraine de fond würmienne. 4 Moraines latérales d'un stade de retrait. 5 Graviers de la terrasse supérieure. 6 Graviers de la terrasse inférieure. 7 Cours d'eau actuel. Remarque. Les dépóts glaciaires profonds (Riss) et les terrasses plus récentes que le retrait glaciaire ne figurent pas sur cette coupe.

\section{Conclusions}

La théorie des emboîtements ne peut s'appliquer sans autre à des nappes de graviers qui sont en relation avec des moraines glaciaires. Il faut admettre, contrairement à ce que l'on suppose, que le dépôt de ces graviers sous-glaciaires s'est fait progressivement de bas en haut, à des altitudes croissantes. Nous estimons qu'il y a dans cette nouvelle façon d'envisager les choses la possibilité d'éliminer certaines divergences entre les auteurs qui reconnaissent les traces de quatre glaciations (Günz, Mindel, Riss, Würm) et ceux qui n'en retrouvent que deux (Riss, Würm).

Je dois laisser de côté ici l'intéressante question de savoir si les dépôts glaciaires profonds, ceux du Riss, correspondent bien à une véritable glaciation. Le manque de faune glaciaire à ce niveau, le développement très limité du Riss vers l'aval, peuvent faire supposer qu'il ne s'agit là que d'une phase préliminaire du grand phénomène glaciaire.

D'autre part, il ne faut pas perdre de vue que des déplacements horizontaux et verticaux se sont certainement produits au cours du Quaternaire. Ils ont entraîné des modifications d'altitudes qu'il est pour l'instant difficile d'apprécier, l'étude de ces phénomènes n'en étant qu'à ses débuts.

\section{Index bibliographique:}

1. BECK, P.: Ueber das schweizerische und europäische Pliozān und Pleistozān. Eclogae geol. Helv., Vol. 26, No 2. Bâle 1933.

2. GAGNEBIN, E.: Les invasions glaciaires dans le bassin du Léman. Bull. Lab. Géologie Université de Lausanne, No 58, 1937.

3. GIGNOUX, M.: Géologie stratigraphique. 2e édition. Paris 1936.

4. HEIM, AL.: Geologie der Schweiz, Vol. I. Leipzig 1919.

5. JAYET, A.: Origine et âge de l'alluvion ancienne des environs de Genève. Compte-rendu séances Soc. Phys. et Hist. Nat. de Genève, Vol. 62, No 2. Gendre 1945.

6. - A propos de l'âge du maximum glaciaire quaternaire. Ecl. geol. Helvetiae, Vol. 38, No 2. Bâle 1946

7. - Glaciologie quatemaire et Préhistoire. Archives suisses d'Anthropologie générale. Vol. XI, No 2. Genève 1945.

8. JOUKOWSKY. E.: Géologie et eaux souterraines du Pays de Genève. Genève 1941.

9. MILANKOWITCH, M.: Un chapitre de l'Histoire de la Terre dans la lumière des sciences mathématiques. Revue mathématique de l'Union interbalkanique, II, fasc. 1. Athènes 1938.

10. PAREJAS, E.: Notice explicative de la feuille 12 (Genève) de l'atlas geologique de la Suisse. Berne 1938.

11. PENCK, A., et BRƯCKNER, E.: Dic Alpen im Eiszcitalter. Leipzig 1901-1909. 